Supporting Information

\title{
Facile Synthesis of Semiconductor and Noble Metal Nanocrystals in High-Boiling Two-Phase Liquid/Liquid Systems
}

\author{
Qi-yu Yu, ${ }^{1,2}$ Chun-yan Liu, ${ }^{*}$, Zhi-ying Zhang, ${ }^{1}$ and Yun Liu ${ }^{1}$ \\ ${ }^{1}$ Laboratory of Organic Optoelectronic Functional Materials and Molecular Engineering, Technical \\ Institute of Physics and Chemistry, the Chinese Academy of Sciences, Beijing 100080 \\ ${ }^{2}$ Graduate University of the Chinese Academy of Sciences, Beijing 100049
}

E-mail: cyliu@mail.ipc.ac.cn. 


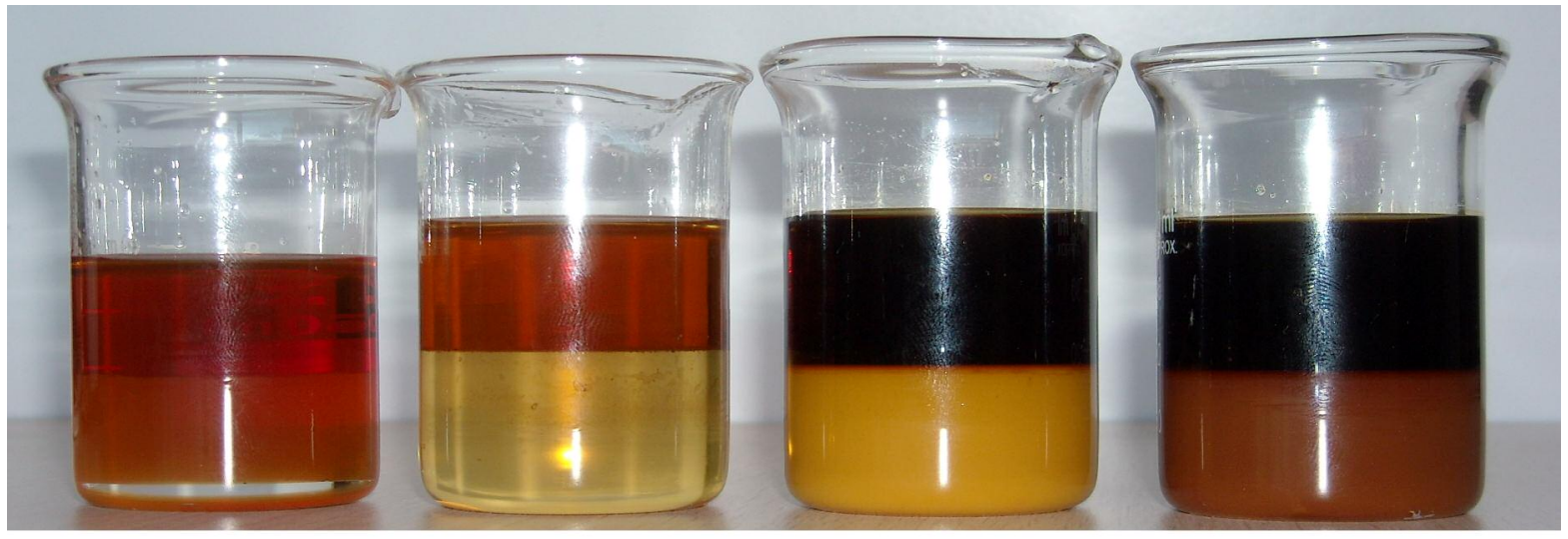

$\begin{array}{llll}\text { (a) } 0 D E / g 1 y c e r o 1 & \text { (b) paraffin/g1ycerol } & \text { (c) ODE/g1ycero1 } & \text { (d) paraffin/g1ycerol }\end{array}$

Figure S1. Typical images of the two-phase mixtures after reaction and being transferred into flasks. (a) and (b): the synthesis of CdS nanocrystals; (c) and (d): the synthesis of Ag nanocrystals. 

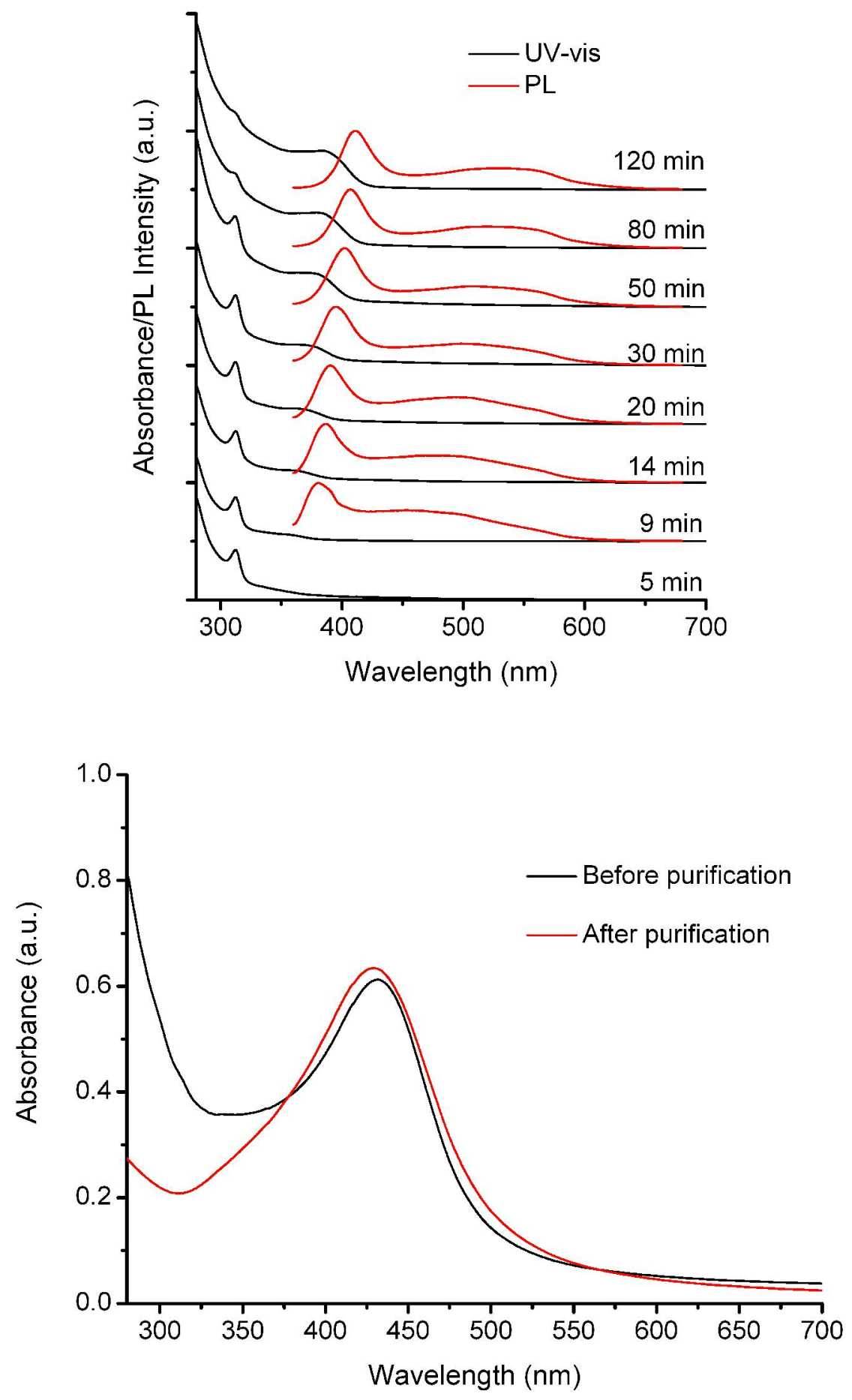

Figure S2. Temporal evolution of UV-vis absorption and PL spectra of the CdS nanocrystals prepared in paraffin/glycerol (top). UV-vis absorption spectra of the Ag colloids prepared in paraffin/glycerol (bottom). 

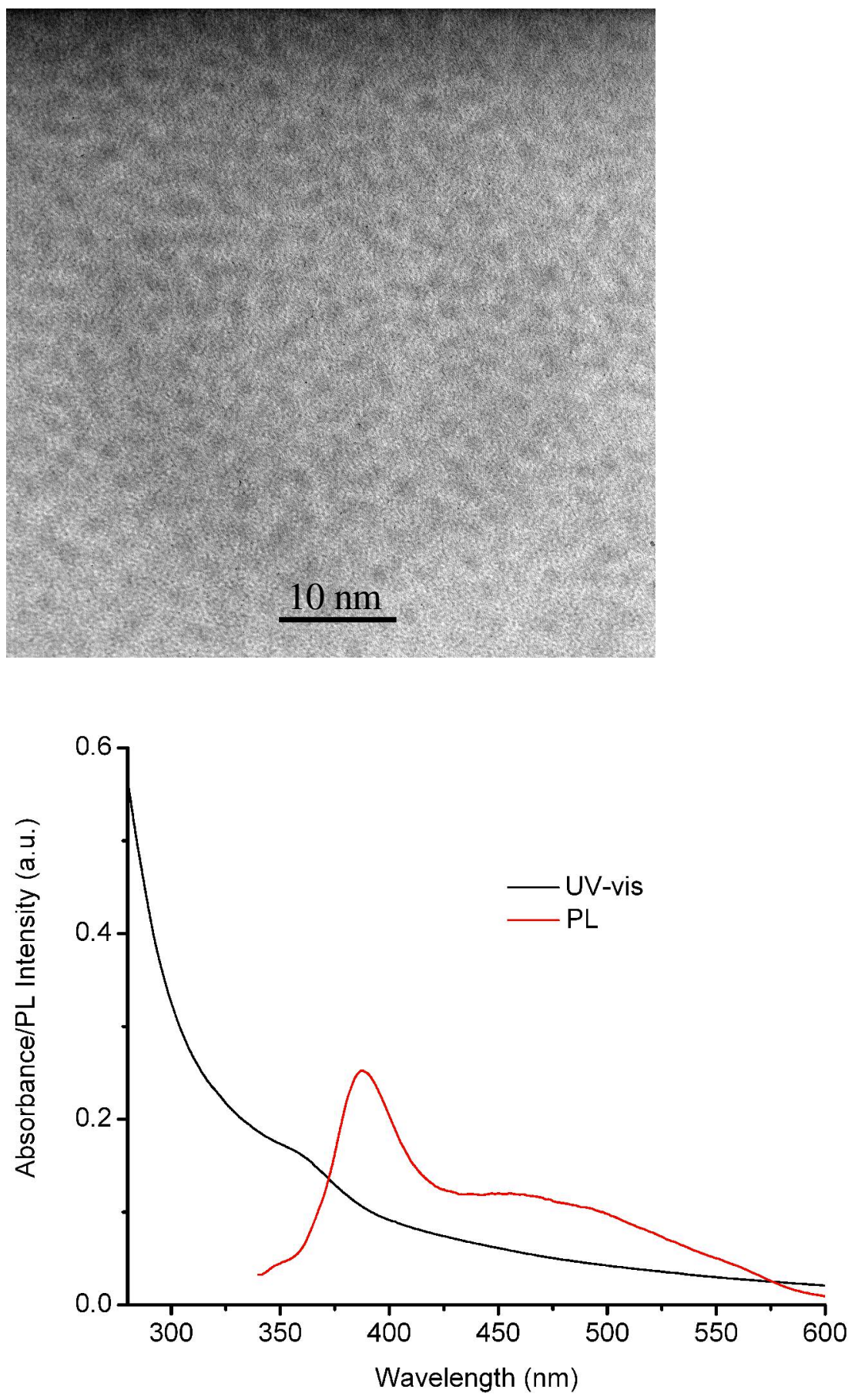

Figure S3. HRTEM image (top) and optical spectra (down) of the CdS nanocrystals prepared in air. The reaction time was 4 hours. TEM observation of this sample was difficult due to extremely small size of the particles, for which the contrast variation under the beam often did not differ sufficiently enough from the normal fluctuations of the carbon support film. 


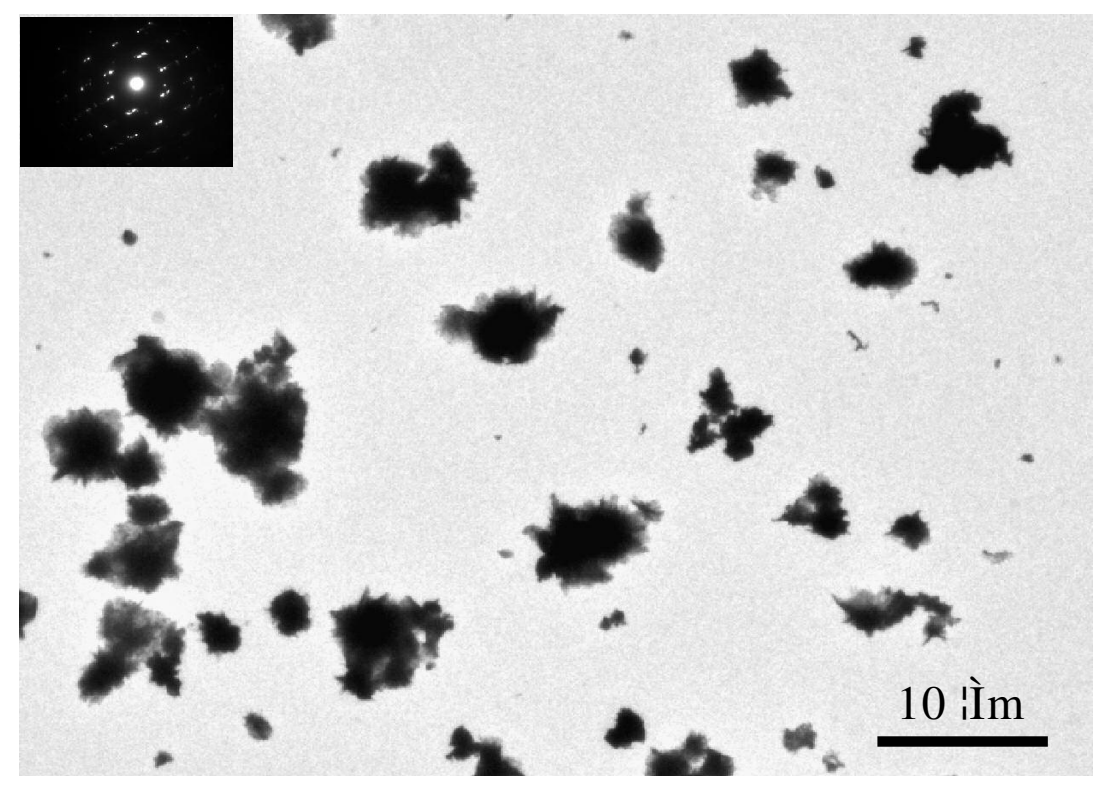

Figure S4. TEM image of Ag crystals made in ODE/water at $90{ }^{\circ} \mathrm{C}$. A selected area electron diffraction pattern is presented in the top left inset. 